\title{
Inattentional blindness: A combination of a relational set and a feature inhibition set?
}

\author{
Rebecca R. Goldstein ${ }^{1}$ - Melissa R. Beck ${ }^{1}$
}

Published online: 21 March 2016

(C) The Psychonomic Society, Inc. 2016

\begin{abstract}
Two experiments were conducted to directly test the feature set hypothesis and the relational set hypothesis in an inattentional blindness task. The feature set hypothesis predicts that unexpected objects that match the to-be-attended stimuli will be reported most. The relational set hypothesis predicts that unexpected objects that match the relationship between the to-be-attended and the to-be-ignored stimuli will be reported the most. Experiment 1 manipulated the luminance of the stimuli. Participants were instructed to monitor the gray letter shapes and to ignore either black or white letter shapes. The unexpected objects that exhibited the luminance relation of the to-be-attended to the to-be-ignored stimuli were reported by participants the most. Experiment 2 manipulated the color of the stimuli. Participants were instructed to monitor the yellower orange or the redder orange letter shapes and to ignore the redder orange or yellower letter shapes. The unexpected objects that exhibited the color relation of the to-beattended to the to-be-ignored stimuli were reported the most. The results do not support the use of a feature set to accomplish the task and instead support the use of a relational set. In addition, the results point to the concurrent use of multiple attentional sets that are both excitatory and inhibitory.
\end{abstract}

Keywords Inattentional blindness $\cdot$ Visual attention

Rebecca R. Goldstein rgolds5@1su.edu

1 Department of Psychology, Louisiana State University, 206 Audubon, Baton Rouge, LA 70803, USA

\section{Introduction}

The visual environment is teeming with complex information that requires a selection system to handle the influx. Selective attention is used to narrow down the information to a manageable size. One way to narrow the information is to use prior knowledge or task goals. For example, if you are searching for a red shirt in your closet, the task goal of "red shirt" can be used to select red information over information of other colors. Using task goals for selectivity involves tuning of the attentional control settings used to complete the task (Folk \& Remington, 1998; Most et al., 2001). The specificity of this tuning remains unclear. For example, the attentional control setting may be for a specific shade of red (a feature set), or it may be for any color redder than another color (a relational set). The degree to which these relative settings can be used and how they may change depending on the task demands is not fully understood. This study examines this question towards the larger goal of understanding the effects of task goals on selective attention.

Selective attention can occur based on tuning to specific features (Duncan \& Humphreys, 1989; Martinez-Trujillo \& Treue, 2004; Treue \& Martinez-Trujillo, 2006;Wolfe, 1994). An attentional control setting could be created for the feature that is the target of a visual search task. For example, a visual search target that is both red and oriented $45^{\circ}$ would result in heightened activity for feature receptors that respond strongly to red information and for receptors that respond strongly to $45^{\circ}$ angles. This increased activity for receptors tuned to specific features would lead to an attentional bias toward both red items and $45^{\circ}$ items. There are two possible matches that could trigger the allocation of attention: (1) items that are the exact shade of red and (2) items that fall within a certain degree of the shade of red. In both cases, the assumption is that the attentional control setting is created for specific 
features. The Feature Set hypothesis predicts that attentional control settings will be created for the critical features and the allocation of attention will be to features in the environment that match the critical feature.

The allocation of attention can also be determined by relationships and contexts within the environment. Becker (2010) postulated that the visual system uses the relationships between items in the environment to create attentional control settings (i.e., relational sets). Becker, Folk, and Remington (2013) reasoned that the stimuli in a visual search task could lead to a relational set based on the relationship between the colors of the targets and the distractors. Therefore, when participants searched for an orange target among gold distractors, a relational set of "redder" may be used. To test this, the visual search trials were preceded by a singleton cue that either matched or did not match the location of the upcoming target. In one condition, the color singleton cue (red) did not match the color of the target (orange), but did match the relationship (redder) of the target to the distractors (gold). If a relational set of redder is used, the red singleton should capture attention and lead to slower responses when the cue is not in the target location and faster responses when it is in the target location. This is what was found, suggesting that the attention system can use the relationships between targets and distractors to bias attention. The Relational Set hypothesis predicts that attentional control settings will be created for relationships between the feature of the task relevant information and the feature of non-relevant information in the environment, and the allocation of attention will be to information in the environment that shares this relationship.

In addition to visual search tasks, attentional control settings have been studied using inattentional blindness tasks (Most, Scholl, Clifford, \& Simons, 2005; Most et al., 2001). In these tasks, participants generally track a subset of items as they move, while ignoring another set of items (Koivisto \& Revonsuo, 2008; Most, Scholl, Clifford, \& Simons, 2005; Most et al., 2001). The two sets of items differ by at least one feature, and while the tracking is occurring, an unexpected object moves across the screen. The degree to which unexpected objects of varying feature similarity of to the to-be-attended and tobe-ignored stimuli are detected is used to measure of the attentional control setting. In a study demonstrating inattentional blindness, some participants were instructed to count the number of passes made by a white shirt team while ignoring passes made by a black shirt team (Simons \& Chabris, 1999). About half of these participants failed to report seeing a black gorilla that walked through the center of the ball toss game. However, participants counting the passes of the black shirt team had no trouble detecting the unexpected object (the gorilla). Simons and Chabris' (1999) study demonstrates that the attention control setting, which is based on the task instructions (count passes of black shirt or white shirt team), determines what irrelevant information is selected and detected.

Inattentional blindness could occur due to attentional control settings based on a specific feature or based on item relationships. Most et al. (2001) examined the role of similarity between information in the environment and attentional control settings in an inattentional blindness task. Participants counted the number of times black letter shapes bounced off the sides of a box while ignoring white letter shapes. On the third trial, one of four possible unexpected objects (UEOs) crossed the screen. The UEO was black (same as to-beattended letter shapes), dark gray, light gray, or white (same as to-be-ignored letter shapes). Most et al. found that the black, dark gray, and light gray UEOs were reported more than the white UEO, and that the rate of noticing the black UEO was higher than the dark gray and light gray UEOs. In this instance, it appears that there is evidence to support both the feature set hypothesis (the UEO matching the target is noticed most) and the relational set hypothesis (the UEOs that were relational matches (darker) were noticed more than the white UEO). However, because the relational match UEOs were between the to-be-attended and to-be-ignored stimuli on the continuum of light to dark luminance, the targets were a better fit for a relational set (darker) than the gray UEOs. Therefore, it is unclear from this experiment if the data support both the feature set and the relational set hypotheses or if the data only support the relational set hypothesis.

In a second experiment, Most et al. (2001) tested a UEO that was a better match for the relational set than the to-beattended stimuli. Participants monitored gray letter shapes with either black or white to-be-ignored letter shapes. The authors found that a black UEO was reported more when the to-be-ignored stimuli were white, and a white UEO was reported more when the to-be-ignored stimuli were black. It was concluded that participants created attentional control settings for "darker luminance" when the distractors were white and for "lighter luminance" when the distractors were black. Most et al. (2001) concluded that the attentional control settings can be based on the relation of the target to the distractors. However, this experiment did not include a UEO that matched the to-be-attended stimuli. It is therefore unknown, as predicted by the feature set hypothesis, if the UEO that matched the to-be-attended stimuli would lead to higher rates of noticing than would be found for a UEO that was a better match for the relationship between the to-be-attended and the to-be-ignored stimuli.

In the present study, we used the inattentional blindness design from Most et al. (2001) and included both UEOs that were best matches for the relationship of the to-be-attended to the to-be-ignored stimuli and UEOs that matched the to-beattended stimuli. Participants tracked gray letter shapes with either black or white to-be-ignored stimuli. The UEO was either gray, black, or white such that it either matched the to- 
be attended stimuli, matched the to-be ignored stimuli, or best matched the relationship of the to-be-attended to the to-beignored stimuli. This allowed us to test whether irrelevant information that best exemplifies the created relationship results in the same or a lower rate of reporting the UEO compared to an exact feature match. According to the relational set hypothesis, an attentional control setting can be created for a relationship or context and any information that matches this relationship will capture attention. It is predicted that when participants monitor gray letter shapes among black letter shapes, both the gray and the white UEOs will be noticed more than the black UEO in support of the relational set hypothesis. In contrast, the feature set hypothesis predicts that irrelevant information that is an exact match to the target (i.e., the gray UEO for the current design) should capture attention the most.

\section{Experiment 1}

\section{Participants}

The number of participants was based on effect size estimates from Most and colleagues (2001). Thirty-six participants were needed per group to achieve an estimated power of .85. 249 Louisiana State University undergraduates participated in this study for course credit. They had a mean age of 19.8 years and reported normal or corrected-to-normal vision. Data included in the analysis comes from 216 participants. Out of the 33 participants excluded from analysis: 11 were excluded for failing to see the unexpected object during the full attention trial, four were excluded for reporting impaired vision, and 18 were excluded for a programming error that causes a letter shape to leave the screen. Participants were randomly assigned to one of six conditions and there were 36 participants in each condition.

\section{Design}

The experiment had a 2 (relation: lighter, darker) $\times 3$ (UEO: to-be-attended match, to-be-ignored match, best relation match) between subjects design (Table 1). Therefore, there were six conditions. For three of the conditions, the to-beignored stimuli were black, so a relational set would be for "lighter," and for the other three conditions the to-be-ignored stimuli where white, so the relational set would be for "darker." The UEO either matched the to-be-attended stimuli (gray) or matched the to-be-ignored stimuli (black when the to-be-ignored stimuli were black or white when the to-beignored stimuli were white), or was a different feature from both the to-be-attended and the to-be-ignored stimuli (black when the to-be-ignored stimuli were white or white when the to-be-ignored were black), but matched the relationship of the to-be-attended to the to-be-ignored (darker and lighter, respectively).

\section{Materials}

Two Apple iMac computers with 20-in. LCD displays with a $1,680 \times 1,050$ resolution and two Apple iMac computers with 21.5-in LCD displays with a resolution set to $1,680 \times 1,050$ presented the stimuli. Psychophysics Toolbox (Brainard, 1997; Pelli, 1997) managed the presentation of the experiment. In a dimly lit room, participants sat approximately $57 \mathrm{~cm}$ from the computer without constraining viewing distance.

On all five trials, a stationary blue $(0,0,255)$ square $(0.25 \times$ $0.25 \mathrm{~cm})$ appeared in the center of an aquamarine $(102,205$, $170)$ box $(12.7 \times 15.5 \mathrm{~cm})$ for $15 \mathrm{~s}$. In addition to the blue square, four $\mathrm{T}$ letter shapes and four L letter shapes $(1 \mathrm{~cm} \times$ $1 \mathrm{~cm}$ ) appeared on screen moving randomly $2-5 \mathrm{~cm} / \mathrm{s}$. Two of the $\mathrm{T}$ letter shapes and two of the L letter shapes appeared in gray $(138,138,138)$, while the other two T shapes and two L shapes appeared in black $(0,0,0)$ or white $(255,255,255)$ depending on the to-be-ignored condition. On the final three trials, a cross $(1 \times 1 \mathrm{~cm})$ appeared on screen at the 5 -s mark moving right to left across the entire screen and disappeared at the 10-s mark (Fig. 1). Depending on the UEO condition, the cross appeared in black, white, or gray.

\section{Procedure}

Instructions present on the screen and read aloud informed the participant to remain focused on the blue square in the center of the screen while silently counting the number of times the gray $\mathrm{T}$ and $\mathrm{L}$ shapes bounced off the sides of the box. Participants always attended to the gray letter shapes, while half of the participants were presented with and instructed to ignore black shapes and half were presented with and instructed to ignore white shapes. The screen prompted participants to enter the silent bounce count using the keyboard immediately following trials one through four.

During the critical trial (trial three) the cross (UEO) appeared for the first time. After participants entered their silent count, the participants answered four questions (from Most et al., 2001) regarding the UEO. The first question read "On the last trial, did you see something other than the black/white and gray letters (anything that had not been present on the first two trials)?" Using the keyboard the participant responded by pressing "Y" for yes or " $\mathrm{N}$ " for no. The remaining three questions focused on the features of the UEO. The first feature question read "If you did see something on the last trial that had not been present during the first two trials, please indicate the color of the objects and indicate if you were guessing." Participants chose from ten statements: five statements to choose one of five colors (e.g., black, white, gray, blue, and 
Table 1 Relationships between to-be-attended, to-be-ignored, and unexpected objects (UEOs) in Experiment 1 and Experiment 2

\begin{tabular}{|c|c|c|c|c|}
\hline \multicolumn{5}{|c|}{ Experiment 1: Luminance } \\
\hline Relationship & To-be-attended & To-be-ignored & UEO & UEO condition \\
\hline \multirow[t]{3}{*}{ Lighter } & Gray & Black & Black & To-be-ignored \\
\hline & Gray & Black & Gray & To-be-attended \\
\hline & Gray & Black & White & Best relation \\
\hline \multirow[t]{3}{*}{ Darker } & Gray & White & Black & Best relation \\
\hline & Gray & White & Gray & To-be-attended \\
\hline & Gray & White & White & To-be-ignored \\
\hline \multicolumn{5}{|c|}{ Experiment 2: Color } \\
\hline Relationship & To-be-attended & To-be-ignored & UEO & UEO condition \\
\hline \multirow[t]{4}{*}{ Redder } & Redder orange & Yellower orange & Red & Best relation \\
\hline & Redder orange & Yellower orange & Redder Orange & To-be-attended \\
\hline & Redder orange & Yellower orange & Yellower Orange & To-be-ignored \\
\hline & Redder orange & Yellower orange & Yellow & Opposite relation \\
\hline \multirow[t]{4}{*}{ Yellower } & Yellower orange & Redder orange & Red & Opposite relation \\
\hline & Yellower orange & Redder orange & Redder Orange & To-be-ignored \\
\hline & Yellower orange & Redder orange & Yellower Orange & To-be-attended \\
\hline & Yellower orange & Redder orange & Yellow & Best relation \\
\hline
\end{tabular}

red) (e.g., "Press 0 if it was black," "Press 1 if it was white") and five statements to guess one of five colors ("Press 5 if it was black and you are guessing," "Press 6 if it was white and you are guessing"). The second feature question read "If you did see something during the last trial that had not been present in the first two trials, please indicate its direction of movement. If you did not see something please guess." Participants chose from eight statements: four statements to choose one of four directions (right, left, up, and down) (e.g., "Press 1 if it was moving right," "Press 2 if it was moving left") and four

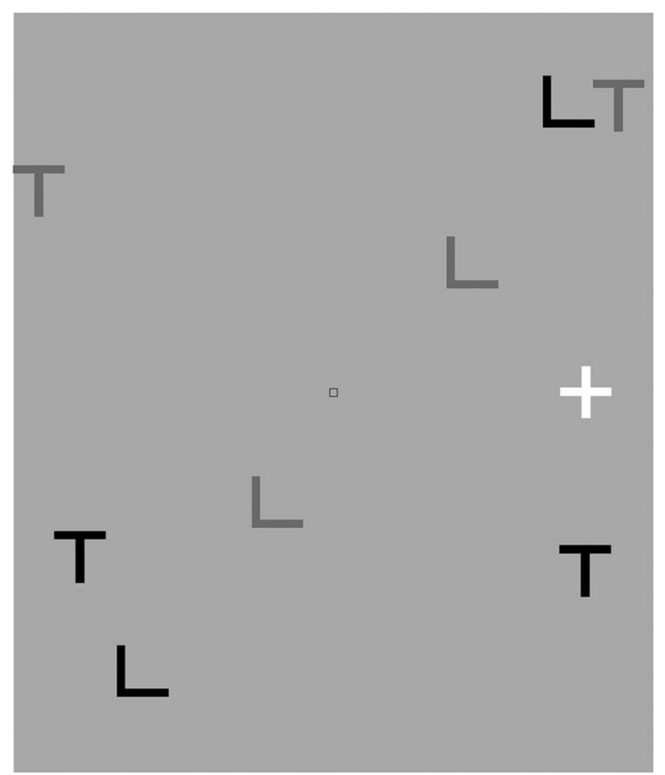

Fig. 1 A grayscale screen shot of the critical trial in Experiment 1 with black to-be-ignored stimuli and a white unexpected object statements to guess one of four directions ("Press 5 if it was moving right and you are guessing," "Press 6 if it was moving left and you are guessing"). The final statement read "If you did see something during the last trial that had not been present during the first two trials, please indicate which shape you saw from the four examples below." A square, triangle, cross, and circle appeared below the statement. The divided attention trial (trial four) followed the same procedure as the critical trial.

Prior to the start of the full attention trial (trial five), instructions appeared on the screen reading "On the next trial you are not required to respond to the gray letter shapes. Simply watch the screen." After trial five ended, participants were asked the four questions described above.

\section{Results}

To be classified as having seen the UEO, a participant had to respond "yes" to the first question and correctly choose one of the three features or respond "no" to the first question and correctly choose all three features. The rate of noticing on the critical trial (i.e., the first trial including the UEO) differed across all the UEOs for the lighter condition, $X^{2}=46.949$ (2, $\mathrm{N}=108), p<.001$, Cramer's $V=.66$, and for the darker condition, $X^{2}=49.871(2, \mathrm{~N}=108), p<.001$, Cramer's $V=.68$ (Fig. 2). Furthermore, the same pattern of results was found on the divided attention trial for the lighter condition, $X^{2}=19.850$ (2, $\mathrm{N}=108), p<.001$, Cramer's $V=.43$, and for the darker condition, $X^{2}=19.516(2, \mathrm{~N}=108), p<.001$, Cramer's $V=.42$.

According to the relational set hypothesis, information that shares the relationship of the to-be-attended to the to-be- 


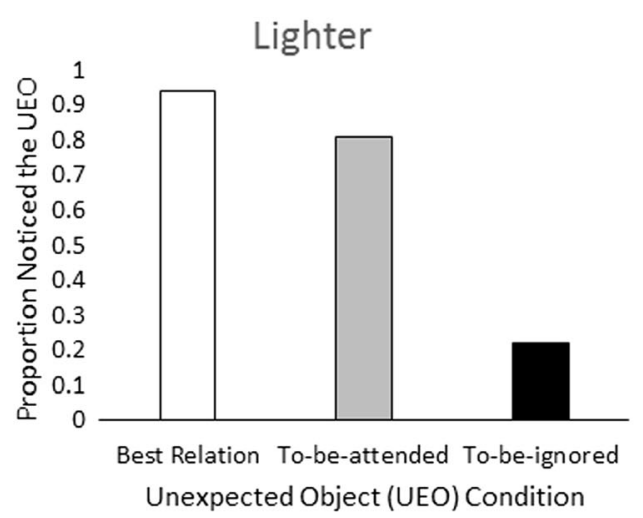

Fig. 2 The proportion of the participants classified as reporting having seen the unexpected object (UEO) in each condition in Experiment 1. The graph on the left shows the proportion of participants that noticed the

ignored stimuli should be noticed most often. Therefore, the rate of noticing the best relation match UEO and the to-beattended match UEO should be higher than the rate of noticing the to-be-ignored match UEO. This was tested using onetailed two-proportion z-tests. The z-tests for the critical trial demonstrated that both UEOs that were consistent with the relationship of the to-be-attended to the to-be-ignored stimuli (best relation match and to-be-attended match UEO) were noticed more than the UEOs that were not consistent with the relationship (to-be-ignored match UEOs). When the tobe-attended stimuli were lighter than the to-be-ignored stimuli (lighter relation condition), both the best relation match UEO $(\mathrm{M}=94.44 \%), z=-6.22, p<.01$, and the to-be-attended match UEO $(\mathrm{M}=80.56 \%), z=-4.95, p<.01$, were noticed more often than the to-be-ignored match UEO ( $\mathrm{M}=22.22 \%)$. When the to-be-attended stimuli were darker than the to-beignored stimuli (darker relation condition), both the best relation match UEO $(\mathrm{M}=88.89 \%), z=-6.14, p<.01$, and the tobe-attended match UEO $(83.33 \%), z=-5.66, p<.01$, were noticed more often than the to-be-ignored match UEO ( $\mathrm{M}=$ $16.67 \%)$.

Also consistent with the relational set hypothesis is the prediction that the UEO that best exemplifies the relationship of the to-be-attended to the to-be-ignored stimuli should be noticed more often or equally often than the to-be-attended match UEO. Alternatively, the feature set hypothesis predicts that the to-be-attended match UEO will be noticed more often than the best relation match UEO. Therefore, the rates of noticing the best relation match UEO and to-be-attended match UEO were submitted to one-tailed two-proportion z-tests. The z-tests for the critical trial in the lighter relation condition demonstrated that the best relation match UEO $(\mathrm{M}=$ $94.44 \%)$ was noticed more often than the to-be-attended match UEO $(\mathrm{M}=80.56 \%), z=1.78, p=.037$. In the darker relation condition, the best relation match UEO $(\mathrm{M}=88.89 \%)$ was noticed at a similar rate to the to-be-attended match UEO $(\mathrm{M}=83.33 \%), z=0.68, p=.248$.

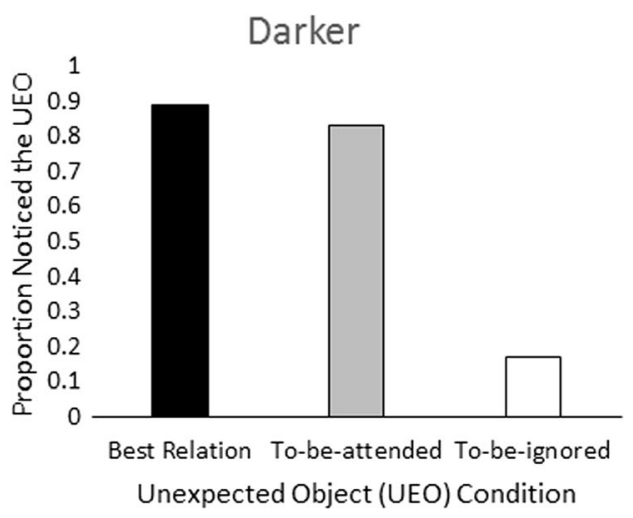

UEO after being instructed to ignore the black $\mathrm{T}$ and $\mathrm{L}$ letter shapes. The graph on the right shows the proportion of participants that noticed the UEO after being instructed to ignore the white $\mathrm{T}$ and $\mathrm{L}$ letter shapes

Absolute bounce error (absolute value of the number of bounces reported minus the actual number divided by the actual number) did not differ for those classified as noticing the UEO and those classified as not noticing the UEO for both the lighter, $t(106)=.813, p=.418, d=.16$, and darker relation conditions, $t(106)=.214, p=.831, d=.04$.

\section{Experiment 2}

The results of Experiment 1 are consistent with Most et al.'s (2001) finding that UEOs that match a broad attentional control setting for "darker luminance" or "lighter luminance" are noticed more often than UEOs that did not match this relational set. Furthermore, the feature set hypothesis was not supported as the to-be-attended match UEO was not noticed more often than the best relation match UEO. Therefore, the data from Experiment 1 support the conclusion that top-down control over the attentional system is not strictly set for feature specifics, but can be set for relations based on luminance of stimuli.

Experiment 2 was designed to expand upon Experiment 1 by testing for attentional control settings based on color relations of stimuli. In Experiment 2, instead of stimuli that only differed in luminance (black, white, and gray), the stimuli had different values around the color wheel (for similar use of color relations see Becker et al., 2013). Specifically, Experiment 2 tested for the ability to use relational sets for "redder" and "yellower." For Experiment 2, the feature set hypothesis predicts the UEO that is an exact color match to the to-be-attended stimuli (e.g., yellower orange UEO with yellower orange to-be-attended letter shapes) will be noticed more than UEOs that do not match the to-be-attended stimuli. The relational set hypothesis predicts the UEOs that match the relationship (e.g., a yellow UEO when there is a yellower relation) of the to-be-attended stimuli (yellower orange) to the to-be-ignored stimuli (e.g., redder orange) will be noticed 
more often than UEOs that do not match the relation (e.g., red UEOs).

Experiment 2 also tested two additional hypotheses that cannot be ruled out by the results from Experiment 1. In Experiment 1, both of the UEOs that exhibited the relationship of darker or lighter luminance were noticed more often than the UEO that matched the to-be-ignored stimuli. However, there were no UEOs in Experiment 1 that did not match the relational set and did not match the distractors. Therefore, it remains possible that participants were inhibiting the to-beignored stimuli, rather than using attentional control settings based on the relationship of the to-be-attended items to the tobe-ignored items. Experiment 2 addressed this by testing the Feature Inhibition Set hypothesis and the Relational Inhibition Set hypothesis.

The feature inhibition set hypothesis predicts that any distractor that does not match the to-be-ignored stimuli will be noticed. Therefore, in Experiment 2 we included a UEO that did not match the relation of the to-be-attended stimuli to the to-be-ignored stimuli and did not match the to-be-ignored stimuli. For example, when participants attended to yellower orange stimuli and ignored redder orange stimuli, there could be a yellow, yellower orange, redder orange, or red UEO. The yellow UEO best matches the relational set of the target to the to-be-ignored stimuli (i.e., the attended stimuli are yellower than the ignored stimuli), and the yellower orange UEO also matches this relational set. However, the red UEO does not match the set and does not match the to-be-ignored stimuli. If the color of the to-be-ignored stimuli is being inhibited, rather than the use of a relational set, then the red UEO (opposite relation match UEO) should be noticed more than the to-beignored match UEO.

According to the relational inhibition set hypothesis, an attentional control setting can occur based on the relationship of the to-be-ignored stimuli to the to-be-attended stimuli rather than the other way around as is predicted by the relational set hypothesis. That is, when participants attended to yellower orange stimuli and ignored redder orange stimuli, the relational set hypothesis would suggest capture by stimuli yellower than the distractors, while the relational inhibition set hypothesis would predict inhibition of stimuli that are opposite the relational match (redder). Therefore, when participants attended to yellower orange stimuli and ignored redder orange stimuli, a red UEO (opposite relation match UEO) would be noticed at a rate similar to or less than the rate of noticing the redder orange UEO (to-be-ignored match UEO).

\section{Participants}

Three hundred and forty-four Louisiana State University undergraduates participated in this study for course credit. They had a mean age of 20 years and reported normal or correctedto-normal vision. Data from 288 participants are included in the analysis. Out of the 56 participants excluded from analysis, 24 were excluded for failing to see the unexpected object during the full attention trial, five were excluded for reporting impaired vision, and 27 were excluded for a programming error that caused a letter shape to leave the screen. Participants were randomly assigned to one of eight conditions and there were 36 participants in each condition.

\section{Design}

The experiment had a 2 (relation: redder, yellower) $\times 4$ (UEO: best relation match, to-be-attended match, to-be-ignored match, opposite relation match) between subjects design (Table 1). Therefore, there were eight conditions. For four of the conditions, the to-be-attended stimuli (yellower orange) were yellower than the to-be-ignored stimuli (redder orange), and for the other four conditions the to-be-attended stimuli (redder orange) were redder than the to-be-ignored stimuli (yellower orange). The to-be-attended match UEO was redder orange when the to-be-attended stimuli were redder orange, and it was yellower orange when the to-be attended stimuli were yellower orange. The to-be-ignored match UEO was redder orange when the to-be-attended stimuli were yellower orange and the to-be-ignored match UEO was yellower orange when the to-be-attended stimuli were redder orange. For the redder orange to-be-attended stimuli, the best relation match was red because the redder orange to-be-attended stimuli were redder than the yellower orange to-be-ignored stimuli. The yellow UEO was the opposite relation match when the to-be-attended stimuli were redder orange (a "redder" attentional set relationship) because it matched the opposite of the attentional set relationship. The red UEO was the opposite relation match UEO when the to-be-attended stimuli were yellower orange (a "yellower" attentional set relationship) because it matched the opposite of the attentional set relationship.

\section{Materials}

The materials are the same as for Experiment 1 except for the following: Two Apple iMac computers with 21.5-in. LCD displays with the resolution set to $1,680 \times 1,050$ presented the stimuli. The $\mathrm{T}$ and $\mathrm{L}$ letter shapes that moved around in the gray $(138,138,138)$ box appeared as yellower orange $(255,140,0)$ and redder orange $(255$, $76,0)$. The instructions distinguished the to-be-attended stimuli from the to-be-ignored stimuli (e.g., track the yellower orange letter shapes, ignore the redder orange letter shapes). On the critical trial, the unexpected object color could be red $(255,0,0)$, redder orange, yellower orange, or yellow $(255,200,0)$. 


\section{Procedure}

The procedure is the same as Experiment 1 except for the following: The participants did not all attend to the same color. The instructions informed half the participants to attend to the "light orange" (i.e., yellower orange) letter shapes and the other half to attend to the "dark orange" (i.e., redder orange) letter shapes. On the critical trial and divided attention trial after recording the number of bounces, a prompt appeared on the screen instructing the participants to write down a description of what happened during the trial on a provided sheet of paper (description prompt; Koivisto \& Revonsuo, 2008; Most, 2011; Most et al., 2001). The description prompt also appeared immediately after the full attention trial. Following the description prompt, multiple-choice questions similar to those in Experiment 1, but adapted for the colors used in Experiment 2, were presented. Below the color feature question, participants were presented with five colors (e.g., yellow, redder orange, yellower orange, red, white) for both the yellower and redder conditions.

\section{Results}

To be classified as having seen the UEO, a participant had to respond "yes" to the first multiple-choice question and correctly choose one of the three features or respond "no" to the first question and correctly choose all three features. ${ }^{1}$ The rate of noticing on the critical trial (i.e., the first trial including the UEO) differed across all the UEOs for the yellower condition, $X^{2}=52.819(3, \mathrm{~N}=144), p<.001$, Cramer's $V=.60$, and for the redder condition, $X^{2}=25.341(3, \mathrm{~N}=144), p<.001$, Cramer's $V=.42$ (Fig. 3). Furthermore, rates of noticing also differed on the divided attention trial for the yellower condition, $X^{2}=10.684(3, \mathrm{~N}=144), p=.014$, Cramer's $V=.27$, and for the redder condition, $X^{2}=17.127(3, \mathrm{~N}=144), p=.001$, Cramer's $V=.34$.

\footnotetext{
${ }^{1}$ The description prompt (i.e., "On the last trial, did you see something other than the light orange and dark orange letters, anything that had not been present on the first two trials)?" was also used to classify the participants as having noticed the UEO. The written answers produced the same pattern of results as the multiple choice answers (critical trial: yellower condition $X^{2}=45.59(3, \mathrm{~N}=144), p<.001$ and redder $X^{2}=$ $15.11(3, \mathrm{~N}=144), p=.002)$. It should be noted that the number of participants classified as noticing the UEO was higher when the multiple choice questions were used to classify compared to when the written answer to the description prompt was used. In the redder condition: 19 written compared 27 multiple choice for the red UEO, 16 written and 24 multiple choice for the redder orange UEO, six written and eight multiple choice for the yellower orange UEO, 21 written and 24 multiple choice for the yellow UEO. In the yellower condition: eight written and 12 multiple choice for the red UEO, three written and four multiple choice for the redder orange condition, 27 written and 30 multiple choice for the yellower orange condition, 23 written and 28 multiple choice for the yellow condition.
}

Feature set and relational set hypotheses

According to the relational set hypothesis, the rate of noticing the best relation match UEO and the to-be-attended matched UEO should be higher than the rate of noticing the to-beignored match UEO. The one-tailed two-proportion z-tests for the critical trial demonstrated that both UEOs that were consistent with the relationship of the to-be-attended to the tobe-ignored stimuli (best relation match and to-be-attended match UEO) were noticed more than the UEOs that were not consistent with the relationship (to-be-ignored match UEOs). When the to-be-attended stimuli were yellower than the to-be-ignored stimuli (yellower relation condition), both the best relation match UEO $(\mathrm{M}=77.78 \%), z=5.69, p<.01$, and the to-be-attended match UEO $(\mathrm{M}=88.33 \%), z=6.14, p$ $<$.01, were noticed more often than to-be-ignored match UEO $(\mathrm{M}=11.11 \%)$. When the to-be-attended stimuli were redder than the to-be-ignored stimuli (redder relation condition), both the best relation match UEO $(\mathrm{M}=75 \%), z=4.48, p<.01$, and the to-be-attended match UEO (M=66.67\%), $z=3.79, p<$ .01 were noticed more often than the to-be-ignored match UEO $(\mathrm{M}=22.22 \%)$.

The relational set hypothesis predicts that the UEO that best exemplifies the relationship of the to-be-attended to the to-be-ignored stimuli should be noticed more often or equally than the to-be-attended match UEO, while the feature set hypothesis predicts that the to-be-attended match UEO will be noticed more often than the best relation match UEO. The one-tailed two-proportion z-tests for the critical condition demonstrate that the UEOs that best matched the relationship of the to-be-attended to the to-be-ignored stimuli were noticed at a similar rate to the to-be-attended match UEOs. When the to-be-attended stimuli were yellower than the to-be-ignored stimuli (yellower relation condition), the best relation match UEO ( $\mathrm{M}=77.78 \%)$ was noticed just as often as the to-beattended match UEO ( $\mathrm{M}=83.33 \%), z=-0.59, p=.274$. When the to-be-attended stimuli were redder than the to-beignored stimuli (redder relation condition), the best relation match UEO ( $\mathrm{M}=75 \%)$ was noticed just as often as the tobe-attended match UEO $(\mathrm{M}=66.67 \%), z=0.778, p=0.218$.

\section{Feature inhibition and relational inhibition set hypotheses}

According to the relational inhibition set hypothesis, information that shares the relationship of the to-be-ignored to the tobe-attended stimuli should be reported as noticed least often. Therefore, the rate of noticing the opposite relation match UEO should be less than or equal to the rate of noticing the to-be-ignored match UEO. This was tested using one-tailed two-proportion z-tests. The z-tests for the critical trial demonstrated that the UEOs that were the best match with the relationship of the to-be-ignored to the to-be-attended stimuli (opposite relation match UEO) were noticed more than the UEOs 


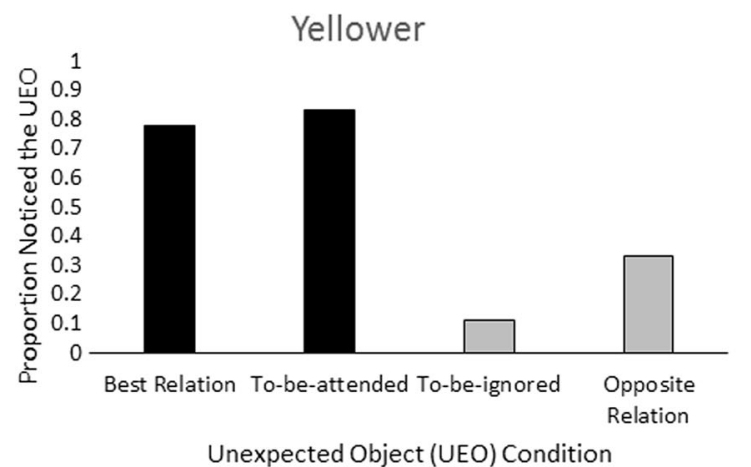

Fig. 3 The proportion of the participants classified as reporting having seen the unexpected object (UEO) in each condition in Experiment 2. In the graph to the left is the proportion of participants that noticed the UEO

that were matches to the to-be-ignored stimuli (to-be-ignored match UEO). When the to-be-ignored stimuli were redder than the to-be-attended stimuli (yellower relation condition), the opposite relation match UEO ( $\mathrm{M}=33.33 \%)$ was noticed more often than the to-be-ignored match UEO $(\mathrm{M}=11.11 \%)$, $z=2.03, p=.021$. When the to-be-ignored stimuli were yellower than the to-be-attended stimuli (redder relation condition), the opposite relation match UEO ( $\mathrm{M}=66.67 \%$ ) was noticed more often than the to-be-ignored match UEO ( $\mathrm{M}=$ $22.22 \%), z=-3.79, p<.01$.

Absolute bounce error (absolute value of the number of bounces reported minus the actual number divided by the actual number) did not differ for those classified as noticing the UEO and those classified as not noticing the UEO for both the yellower, $t(142)=1.566, p=.120, d=.26$ and redder relation conditions, $t(142)=-.661, p=.509, d=.11$.

\section{Discussion}

In Experiment 2, the UEOs that shared the relationship of the to-be-attended to the to-be-ignored stimuli were reported as being noticed most often. This is in line with the attention system being set for relations. In addition, the data do not support the prediction from the feature set hypothesis that the information that is an exact match will be noticed the most often. Similar to the darker condition in Experiment 1, there were no differences between the items that fit the relational set (i.e., redder or yellower). Therefore, consistent with Experiment 1, Experiment 2 supports the relational set hypothesis.

The feature inhibition set and the relational inhibition set hypotheses were also tested in Experiment 2. The findings suggest an attentional control setting was established to inhibit the to-be-ignored stimuli. This was supported by a difference in the number of participants that reported the to-be-ignored match UEO and the opposite relation match UEO. The opposite relation match UEOs were noticed more. It appears that in
Redder

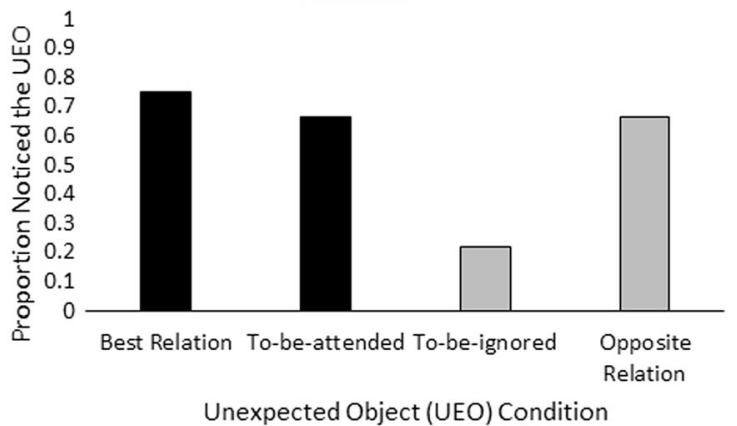

after being instructed to track the yellower orange T and L letter shapes. In the graph to the right is the proportion of participants that noticed the UEO after being instructed track the redder orange $\mathrm{T}$ and $\mathrm{L}$ letter shapes

addition to using a relational set, participants were inhibiting the distractor feature.

\section{General discussion}

The current study is the first to test the feature set and relational set hypotheses in an inattentional blindness task. The feature set hypothesis predicts that top-down control by attentional control settings involves feature-specific control settings. Thus, only items that are exact matches to these settings lead to processing of an item. The relational set hypothesis predicts top-down control by attentional control settings can involve relationship control settings. Thus, items that have the same relationship as these settings lead to processing of an item. Both Experiment 1 and Experiment 2 supported the relational set hypothesis. All the items that exhibited the relationship of the to-be-attended to to-be-ignored stimuli were noticed more than the to-be-ignored stimuli. These results are consistent with previous findings that attentional control settings can be broad and these broad settings are relational (Becker, 2010; Becker et al., 2013).

Furthermore, these results are a continuation and extension of Most et al.'s (2001) findings. Across Most et al.'s Experiments 1 and 2, the best relation match UEO was either also the to-be-attended stimuli (Experiment 1) or was not the to-be-attended stimuli (Experiment 2). Therefore, best relation match UEOs that had and did not have the same feature as the to-be-attended stimuli were never directly compared to each other. In Experiment 1 of Most et al. (2001), the best relational match UEO had the same feature as the to-be-attended stimuli and was noticed more (e.g., $94 \%$ and $94 \%$ ) than UEOs that fell between the to-be-attended and the to-be-ignored stimuli (e.g., $75 \%, 56 \%$ and $44 \%, 12 \%$ ). From those results it is unclear whether the UEO was noticed more because it was an exact feature match or a best relation match. In Experiment 2 of Most et al. (2001) the best relation match UEO did not match the to-be-attended items, but was noticed the most, 
but a UEO that was an exact feature match for the to-beattended stimuli was not included. So it is unclear whether an exact feature match UEO would be noticed more. In the current experiment, the rates of noticing a best relation match UEO and a UEO that was an exact match to the to-be-attended stimuli were compared. The current findings replicate Most et al.'s finding that attentional control settings can be created for relation/categories and do not support the use of an attentional control setting for exact feature match. More importantly, the current results extend Most et al. by finding relational attentional control settings with color, which has only previously been shown in tasks with transient shifts of attention (Becker, 2010; Becker et al., 2013), and by suggesting a feature inhibition attentional control setting can be used in conjunction with a relational attentional control setting.

A possible alternative explanation for the findings is that attention is directed to any items that are distinct in the visual environment. The distinctiveness hypothesis predicts that items with unique values will capture attention (Most et al. 2001; Most, Scholl, Clifford, \& Simons, 2005). Therefore, any UEO that is not the color of the to-be-attended or the to-be-ignored stimuli would capture attention. However, our results do not support this as the sole explanation for several reasons. First, UEOs that were not distinct captured attention. In both experiments, the tobe-attended UEOs were noticed at high rates and these UEOs were not distinct. Second, not all distinctive UEOs captured attention at a high rate. Specifically, the opposite relation UEO in the yellower condition (red UEO) was only noticed at a rate of $33 \%$. Finally, distinct items were noticed at differing rates when they were best relation match UEOs versus when they were opposite relation UEOs even though the distinctiveness in these situations had not changed. Specifically, the rate of noticing the opposite relation UEO in the yellower condition (red $\mathrm{UEO}, 33 \%$ ) was lower than the rate of noticing the best relation UEO in the redder condition (red UEO, $75 \%$ ) (one-tailed two-proportion z-test: $z=3.55, p<.01$ ). In both cases the UEO was the same color and had the same distinctiveness as compared to the other items on the screen. There is some evidence for a role of distinctiveness for the yellow UEO, which was noticed at a high rate both when it was the best relation match UEO and when it was the opposite relation match UEO. However, this was only true for the critical trial and not for the divided attention trial. Once participants were made aware that the UEO would appear on screen, the role of the attentional set is reduced and the rate of noticing the distinct UEOs is similar across color and condition (yellow UEO in redder condition $=83 \%$, yellow UEO in yellower condition $=92 \%$, red UEO in yellower condition $=83 \%$, and red UEO in redder condition $=97 \%$ ). Therefore, although there may be some instances where it appears that distinctiveness can account for the results, the relational set hypothesis and the feature inhibition hypothesis give a more complete account for the results.

The current study is the first to test the feature set hypothesis and relational set hypothesis for detection of unexpected stimuli in a sustained attention task. Previous studies have demonstrated relational sets in visual search tasks (Becker, 2010; Becker, Folk, \& Remington, 2010, 2013). In the visual search tasks that have been used to show the role of a relational set over a feature set, attention is shown to be drawn to items that share the relation. However, there is no measurement of the degree to which the attended items reach awareness. The current study demonstrates that not only can relationconsistent stimuli capture attention, but participants are aware of these stimuli and can later report them accurately. Furthermore, the current results demonstrate that relational sets can be used to sustain attention over time (5,000-15, $000 \mathrm{~ms})$ rather than for just brief visual search tasks ( $\sim 300 \mathrm{~ms})$. Therefore, the current results expand previous research by showing that relational sets are used to sustain attention and can lead to attention capture and awareness of relation-consistent stimuli.

The current results also demonstrate the use of a relational set for sustaining attention across moving stimuli. Du and Jiao (2015) propose an attentional intensive task, such as multiple object tracking, would prove difficult for working memory to extract feature relations. The authors used a rapid serial visual presentation task to demonstrate that relational attentional control settings can only be extracted when the information is central (within the stream) and not when it is peripheral (outside the stream). Feature sets were found to capture attention when central and peripheral. With multiple object tracking the information is moving such that it can be central (in the window), peripheral (outside the window), central again, and so on. The current study demonstrates the extraction of relational attentional control settings from moving objects with the higher rate of reporting the relationship match UEOs compared to the to-be-ignored UEOs in both Experiment 1 and Experiment 2. In addition, it suggests a grouping of relation/categorical information to monitor multiple moving objects at one time.

In sum, our data are consistent with utilizing both a relational attentional control setting and a feature inhibition attentional control setting. The results are consistent with the relational set hypothesis from previous findings (Becker, 2010; Becker, et al., 2013), supporting that relations can be extracted and utilized to complete task goals. The findings further support relational attentional control settings and feature inhibition control settings are not necessarily mutually exclusive. Thus, the current study provides support for use of multiple concurrent mechanisms for the allocation of selective attention. Future research should investigate these differences in 
tuning specificity for attending to-be-attended items and inhibiting to-be-ignored items.

\section{References}

Becker, S. I. (2010). The role of target-distractor relationships in guiding attention and the eyes in visual search. Journal of Experimental Psychology: General, 139, 247-265. doi:10.1037/a0018808

Becker, S. I., Folk, C. L., \& Remington, R. W. (2010). The role of relational information in contingent capture. Journal of Experimental Psychology: Human Perception and Performance, 36, 1460-1476. doi: $10.1037 / \mathrm{a} 0020370$

Becker, S. I., Folk, C. L., \& Remington, R. W. (2013). Attentional capture does not depend feature similarity, but on target-nontarget relations. Psychological Science, 24, 634-647. doi:10.1177/ 095679761245828

Brainard, D. H. (1997). The psychophysics toolbox. Spatial Vision, 10, 433-436.

Du, F., \& Jiao, J. (2016). Feature-based attention is functionally distinct from relation-based attention: The double dissociation between color-based capture and color-relation-based capture attention. Journal of Experimental Psychology: Human Perception and Performance, 42, 480-493. doi: 10.1037/xhp0000165

Duncan, J., \& Humphreys, G. W. (1989). Visual search and stimulus similarity. Psychological Review, 96, 433-458.

Folk, C. L., \& Remington, R. (1998). Selectivity in distraction by irrelevant featural singletons: Evidence for two forms of attentional capture. Journal of Experimental Psychology: Human Perception \& Psychophysics, 24, 847-858.

Koivisto, M., \& Revonsuo, A. (2008). The role of unattended distractors in sustained inattentional blindness. Psychological Research, 72, 39-48. doi:10.1007/s00426-006-0072-4

Martinez-Trujillo, J. C., \& Treue, S. (2004). Feature-based attention increases the selectivity of population responses in primate visual cortex. Current Biology, 14, 744-751. doi:10.1016/j.cub.2004.04. 028

Most, S. B. (2011). Setting sights higher: Category level-attentional set modulates sustained inattentional blindness. Psychological Research, 77, 139-46. doi:10.1007/s00426-011-0379-7

Most, S. B., Scholl, B. J., Clifford, E. R., \& Simons, D. J. (2005). What you see is what you set: Sustained inattentional blindness and the capture of awareness. Psychological Review, 112, 217-242. doi:10. 1037/0033-295X.112.1.217

Most, S. B., Simons, D. J., Scholl, B. J., Jimenez, R., Clifford, E., \& Chabris, C. F. (2001). How not to be seen: The contribution of similarity and selective ignoring to sustained inattentional blindness. Psychological Science, 12, 9-17.

Pelli, D. G. (1997). The videotoolbox software for visual psychophysics: Transforming numbers into movies. Spatial Vision, 10, 437-442.

Simons, D. J., \& Chabris, C. F. (1999). Gorillas in our midst: Sustained inattentional blindness for dynamic events. Perception, 28, 1059 1074.

Treue, S., \& Martinez-Trujillo, J. C. (2006). Visual search and single-cell electrophysiology of attention: Area MT, from sensation to perception. Visual Cognition, 14, 898-910. doi:10.1080/ 13506280500197256

Wolfe, J. M. (1994). Guided Search 2.0: A revised model of visual search. Psychonomic Bulletin \& Review, 1, 202-238. 